\title{
THE WINDING OF A RELAXED ELASTIC LINE ON A CYLINDER*
}

\author{
BY \\ (GERALD S. MANNING ${ }^{1}$ \\ Rutgers, The State University of New Jersey
}

\begin{abstract}
We analyze several cases of the boundary-value problem that determines the path of a relaxed elastic line on a cylinder. A short line with oblique initial tangent deviates from the corresponding helix toward the direction of the axis of the cylinder. A line initially directed along the base circle continues to wind circumferentially if the ratio of its length to the radius of the cylinder does not exceed the critical value $\pi / 2^{3 / 2}$; longer lines deviate from the circle. Infinitely long elastic lines wind in proportion to the logarithm of their arc lengths, as distinct from the direct proportion of a helix. Possible implications for biological structures are discussed.
\end{abstract}

1. Introduction. We recall from an earlier paper [1] the definition of a relaxed elastic line as an arc with fixed initial point and direction and minimum elastic energy $\int_{0}^{l} \kappa^{2}(s) d s$, where $s$ is arc length, $\kappa(s)$ the curvature of the arc, and $l>0$ the length $\int_{0}^{l} d s$ of the arc. If the elastic line is constrained to a curved surface, it generally cannot be straight when relaxed, because $\kappa(s)$ cannot vanish along its length. The relaxed path is determined by the solution of a variational problem with boundary conditions determined by the specified position and direction at $s=0$ and the freedom of the line at $s=l[1,2]$.

We showed in [1] that the boundary-value problem for a general surface is solved for a plane or sphere by the arc of a geodesic, thus confirming the obvious statement that an elastic line relaxed on a plane is straight but lies along a great circle if confined to a sphere. We also showed that the relaxed path is not geodesic (i.e., not a helix) if the surface is a cylinder. A proof that the plane and sphere are the only two surfaces with geodesic relaxed elastic lines may be found in [2]. This result is not obvious. Indeed, in the limit $l \rightarrow 0$, a relaxed elastic line on any surface is geodesic [1]. To bend away from the geodesic curve at any point thus requires energy. The behavior of a short (but nonzero) elastic line is therefore not clear in advance of the calculations. One of the results in this

\footnotetext{
* Received March 3, 1987.

This research has been partially supported by NIH Grant GM 36284-01.

${ }^{1}$ Department of Chemistry, Rutgers University, New Brunswick, New Jersey 08903
} 
paper is that a short elastic line initially directed along the base circle of a cylinder continues to wind along the circle, but a short line directed obliquely does not wind along the corresponding helix. One aspect of the qualitative behavior of a long elastic line, on the other hand, is clear. A long line will tend toward the principal direction of minimum curvature; whatever increase in energy accompanies the local deviation from a geodesic is then more than compensated by the decreased energy along most of the length of the rod.

We examine here some solutions for a cylinder of the boundary-value problem posed in [1] for a general surface. The principal direction of greatest normal curvature on a cylinder is along the base circle, and we will refer to the circumferential direction. The axial direction is understood to be that of the axis of the cylinder and represents the direction of least curvature. The nucleosome core particle in chromosomes provides biophysical motivation, as discussed in [1]. Considerations on this structure, suggested by our result that an elastic line relaxes on a cylinder by tending strongly toward the axial direction, are presented.

2. Boundary-value problem for the cylinder. The path of a relaxed elastic line on a right circular cylinder, with initial direction fixed, is a solution of the following system of equations [1], wherein $u(s)$ is the coordinate value at $s$ that measures distance along the base circle of radius $R, v(s)$ is the distance along an axially directed generator, $\lambda(s)$ is a Lagrange multiplier, and a prime denotes differentiation with respect to $s$,

$$
\begin{aligned}
u^{\prime \prime \prime}-u^{\prime}\left(\lambda+2 R^{-2} u^{\prime 2}\right) & =0, \\
v^{\prime \prime \prime}-\lambda v^{\prime} & =0, \\
u^{\prime 2}+v^{\prime 2} & =1 .
\end{aligned}
$$

Equations (1) and (2) are Euler-Lagrange equations, while Equation (3) recognizes that $s$ is arc length. Both $v$ and $\lambda$ are readily eliminated to yield an equation for the $u$-coordinate. With the assumption that $u^{\prime}$ is nowhere equal to \pm 1 , we get

$$
u^{\prime \prime \prime}+u^{\prime} u^{\prime \prime 2} /\left(1-u^{\prime 2}\right)-2 R^{-2} u^{\prime 3}\left(1-u^{\prime 2}\right)=0 .
$$

We introduce the dimensionless quantities $\sigma=s / l$ and $\mu(\sigma)=u / l$, where $l$ is the fixed length of the elastic line. With the convention that a prime on a Greek letter means differentiation with respect to $\sigma$ (as distinct from a prime on a roman letter, which means differentiation with respect to $s$ ), we can rewrite Eq. (4),

$$
\gamma^{\prime \prime}+\gamma \gamma^{\prime 2} /\left(1-\gamma^{2}\right)=2 r^{2} \gamma^{3}\left(1-\gamma^{2}\right)
$$

where $\gamma=\mu^{\prime}$, and $r=l / R$, the constant ratio of the length of the elastic line to the radius of the cylinder.

We have to specify two boundary conditions for the second-order equation (5) for $\gamma(\sigma)$. The first,

$$
\gamma(0)=a
$$

reflects the initial direction of the elastic line. The extreme values of $a$ are zero for an initially axial direction, and +1 , when the curve initially proceeds in a counterclockwise circumferential direction. The second boundary condition,

$$
\gamma^{\prime}(1)=0, \quad \gamma(1) \neq \pm 1
$$


is obtained from Eq. (40) of [1] by eliminating $v^{\prime \prime}$ (with the help of Eq. (3) above) and corresponds to a free end.

The solution is obvious if the initial direction is axial. Indeed, if $a=0$, the function $\gamma(\sigma)=0$, corresponding to a generator (straight line), solves Eq. (5) and the boundary conditions at both ends.

3. Short elastic line with initially oblique direction. We look first at the case of small $r$. The reader may wish to have in mind the picture of a short elastic line (small $l$ ) on a piece of cylindrical surface formed by slight bending of a plane (large $R$ ). We will solve Eq. (5) by a second-order perturbation procedure.

Let

$$
\gamma(\sigma)=\gamma_{0}(\sigma)+r \gamma_{1}(\sigma)+r^{2} \gamma_{2}(\sigma)+\cdots .
$$

Equations (6) and (7) imply

$$
\begin{array}{lll}
\gamma_{0}(0)=a, & \gamma_{1}(0)=0, & \gamma_{2}(0)=0, \\
\gamma_{0}^{\prime}(1)=0, & \gamma_{1}^{\prime}(1)=0, & \gamma_{2}^{\prime}(1)=0 .
\end{array}
$$

Substitution of Eq. (8) into Eq. (5), followed by independent treatment of the coefficients of the various powers of $r$, yields a hierarchy of equations of the form

$$
\begin{aligned}
\gamma_{0}^{\prime \prime}+\gamma_{0} \gamma_{0}^{\prime 2} /\left(1-\gamma_{0}^{2}\right) & =0 \\
\gamma_{1}^{\prime \prime} & =\gamma_{0}^{\prime} f\left(\gamma_{0}, \gamma_{0}^{\prime}, \gamma_{1}, \gamma_{1}^{\prime}\right), \\
\gamma_{2}^{\prime \prime} & =2 \gamma_{0}^{3}\left(1-\gamma_{0}^{2}\right)+g\left(\gamma_{0}, \gamma_{0}^{\prime}, \gamma_{1}, \gamma_{1}^{\prime}, \gamma_{2}, \gamma_{2}^{\prime}\right),
\end{aligned}
$$

where the functions $f$ and $g$ are well-behaved except for the extreme case $a=1$, which will be discussed separately. The solution of Eq. (10) that satisfies Eqs. (9) is $\gamma_{0}=a$. Substitution into Eq. (11) gives $\gamma_{1}^{\prime \prime}=0$, from which $\gamma_{1}=0$ in order to satisfy Eqs. (9). The function $g$ in Eq. (12) is composed of several terms, each of which is proportional to $\gamma_{0}^{\prime}$ or $\gamma_{1}^{\prime}$, hence vanishes. Thus, $\gamma_{2}^{\prime \prime}=2 a^{3}\left(1-a^{2}\right)$, from which, with Eqs. (9), $\gamma_{2}=-a^{3}(1-$ $\left.a^{2}\right) \sigma(2-\sigma)$. To second order in $r$, then

$$
\gamma(\sigma)=a-r^{2} a^{3}\left(1-a^{2}\right) \sigma(2-\sigma) .
$$

Since $\gamma=\mu^{\prime}$, we can get $\mu(\sigma)$ by integration and then convert to the original coordinate function $u(s)=l \mu$. The axial coordinate $v(s)$ is obtained by application of Eq. (3). The result, if the initial point $[u(0), v(0)]=(0,0)$, is

$$
\begin{aligned}
& u(s)=a s\left[1-R^{-2} a^{2}\left(1-a^{2}\right) s\left(l-\frac{1}{3} s\right)\right], \\
& v(s)= \pm \sqrt{1-a^{2}} s\left[1+R^{-2} a^{4} s\left(l-\frac{1}{3} s\right)\right] .
\end{aligned}
$$

The upper sign is chosen if the elastic line is initially directed in a right-handed sense; the lower sign applies to the left-handed sense.

In agreement with the general considerations in [1], the relaxed elastic line approaches the geodesic corresponding to the initial direction, in this case the helix $u=a s, v=$ $\pm \sqrt{1-a^{2}} s$, if its length $l$ is sufficiently short. If, however, longer elastic lines are considered, their relaxed trajectories depart increasingly toward the axial direction from 
the corresponding helix; the rate of increase of the circumferential coordinate $u(s)$ slows, while the axial coordinate $v(s)$ proceeds at a faster rate to maintain Eq. (3).

The case $a=0$ is represented, according to Eqs. (14) and (15), by the generator $u=0$, $v= \pm s$, while $a=1$ gives an arc along the base circle, $u=s, v=0$. The latter case is delicate, however. Although there is no hint in Eqs. (14) and (15) of a problem at $a=1$, we have already noted that both the differential equation (4) and the boundary equation (7) are valid only if $u^{\prime} \neq 1$. We will now discuss the case of an initially circumferential direction and get an interesting result.

4. Initially circumferential direction. We return to the original system of Eqs. (1-3) and eliminate $\lambda(s)$ and $u(s)$,

$$
v^{\prime \prime \prime}+v^{\prime} v^{\prime \prime 2} /\left(1-v^{\prime 2}\right)+2 R^{-2} v^{\prime}\left(1-v^{\prime 2}\right)^{2}=0 .
$$

Now we may investigate solutions lying near $u(s)=s$, since, for them, $v^{\prime}(s)$ is close to zero (see Eq. (3)), and there is nothing indeterminate in Eq. (16). The boundary conditions are $v(0)=0, v^{\prime}(0)=0, v^{\prime \prime}(l)=0$, the second of which corresponds to the initially circumferential direction of interest in this section, and the third to a free end.

Going over to the dimensionless quantities $\sigma=s / l, \nu(\sigma)=v / l$, and introducing $\omega=\nu^{\prime}$, we get

$$
\omega^{\prime \prime}+\omega \omega^{\prime 2} /\left(1-\omega^{2}\right)+2 r^{2} \omega\left(1-\omega^{2}\right)^{2}=0
$$

with boundary conditions $\omega(0)=0, \omega^{\prime}(1)=0$. Equation (17) and the boundary conditions are satisfied by $\omega=0$. We seek other solutions of Eq. (17) that lie near the base circle of the cylinder. Thus, $\omega$ and its derivatives are small quantities, and Eq. (17) becomes

$$
\omega^{\prime \prime}+2 r^{2} \omega=0
$$

if we neglect terms of third order in smallness. (Note that $r$ in this section is not regarded as either small or large.)

The general solution of Eq. (18) is

$$
\omega=A \sin (\sqrt{2} r \sigma)+B \cos (\sqrt{2} r \sigma)
$$

and the boundary conditions provide two equations for the integration constants $A$ and $B$, from which $B=0$ and

$$
A \cos (\sqrt{2} r)=0 \text {. }
$$

Equation (20) does not determine $A$ uniquely. One solution is $A=0$, and tells us again that a circumferential arc is an equilibrium position, but not necessarily a stable one, of a relaxed elastic line of any length $l$ with circumferential initial direction. But Eq. (20) is also compatible with nonzero $A$ and lengths $l$ such that $\sqrt{2} r=(2 n+1)(\pi / 2)$ for integral $n$. The shortest such length is $l^{*}=\left(\pi / 2^{3 / 2}\right) R \approx 1.11 R$, or about a sixth of the circumference of the base circle. There is an exchange of stabilities at $l^{*}$; shorter elastic lines with circumferential initial direction lie entirely along the base circle, but longer lines become stable only by deviating toward the axial direction. The path of elastic lines of 
length $l>l^{*}$ is obtained by integrating $\omega(\sigma)$. With $v(0)=0$, we get

$$
v(s)=(A / \sqrt{2}) R[1-\cos (\sqrt{2} s / R)] .
$$

It is understood that the approximate validity of Eq. (21) requires $l$ to be not much greater than $l^{*}$, and $A$ not much greater than zero. In fact, the boundary condition $v^{\prime \prime}(l)=0$ for the free end is satisfied only for $l=l^{*}$.

5. The infinitely long elastic line. For large $r$ the picture should be that of a long, narrow cylindrical tube with an elastic line wound around it. Before making approximations, we find it convenient to subject Eq. 5 to the transformation $\gamma=\cos \theta$. In terms of $\theta$, which is the angle by which the elastic line is inclined from the base circle, Eq. (5) becomes

$$
\theta^{\prime \prime}=-2 r^{2} \cos ^{3} \theta \sin \theta
$$

A routine sequence of operations (multiplication of both sides by $\theta^{\prime}$, use of the identity $2 \theta^{\prime} \theta^{\prime \prime}=d \theta^{\prime 2} / d \sigma$, then integration) yields

$$
r^{-1} \theta^{\prime}= \pm\left(\cos ^{4} \theta+c_{1}\right)^{1 / 2}
$$

where $c_{1}$ is a constant of integration. The boundary condition equation (7) is compatible with both $\theta^{\prime}(1)=0$ and $\theta(1)=0$. The latter, however, implies that $\gamma(1)=1$, a third boundary condition on $\gamma(\sigma)$ that overdetermines it, in view of the second-order equation 5. Therefore, $\theta^{\prime}(1)=0$, and the constant of integration $c_{1}$ is identified,

$$
r^{-1} \theta^{\prime}(\sigma)=\left[\cos ^{4} \theta(\sigma)-\cos ^{4} \theta(1)\right]^{1 / 2}
$$

The positive sign has been chosen because, from Eq. (24), $\cos \theta(\sigma)$ must not be less than $\cos \theta(1)$, so that $\theta^{\prime} \geqslant 0$. Returning to the original unknown $\gamma$ shows that integration of Eq. (24) involves integration of the radical of a sixth-order polynomial, so further progress without recourse to an asymptotic analysis for large $r$ is blocked.

We have completed an analysis for distances $s$ near the end of an elastic line of length $l \gg R$. A simpler procedure suggested by Nickerson provides sufficient insight, however, and we will instead discuss her solution [2]. Equation (24) with $\theta(1)=\pi / 2$ is a first integral of Eq. (22). A second integration gives

$$
\theta(s)=\tan ^{-1}\left[(s / R)+c_{0}\right]
$$

where

$$
c_{0}=\tan \theta_{0}, \quad \theta_{0} \neq \pm \pi / 2
$$

and we have returned to the variable $s=l \sigma$. We now have a solution satisfying the initial boundary condition $\theta(0)=\theta_{0}$; however, it is compatible with the value $\pi / 2$ for $\theta(l)$ if and only if $l$ equals infinity. Equation (25) is therefore the solution of our problem for an infinitely long elastic line. The coordinate functions $u(s)$ and $v(s)$ are obtained by 
integration of $\cos \theta$ and $\sin \theta$, respectively,

$$
\begin{aligned}
& u(s)=R \ln \frac{\frac{s}{R}+c_{0}+\left[1+\left(\frac{1}{R}+c_{0}\right)^{2}\right]^{1 / 2}}{c_{0}+\left(1+c_{0}^{2}\right)^{1 / 2}}, \\
& v(s)=R\left\{\left[1+\left(\frac{1}{R}+c_{0}\right)^{2}\right]^{1 / 2}-\left(1+c_{0}^{2}\right)^{1 / 2}\right\} .
\end{aligned}
$$

Examination of the asymptotic behavior for great distances $s$ along the infinitely long elastic line is particularly instructive. The angle of inclination to the base circle, $\theta(s)$, tends toward $\pi / 2$, and the axial coordinate $v(s) \sim s$. Thus, the path of the relaxed elastic line becomes essentially axial regardless of the initial angle of inclination. However, $\phi(s) \sim \ln (b s)$, where $\phi=u / R$ is the winding angle (number of times the elastic line winds around the axis of the cylinder, $\bmod 2 \pi)$, and $b$ is the constant reciprocal length $2 R^{-1}\left[c_{0}+\left(1+\mathrm{c}_{0}^{2}\right)^{1 / 2}\right]^{-1}$. While striving toward the axial direction, the infinite elastic line winds forever about the cylinder, but at a much slower rate than a helix, which winds in proportion to $s$.

6. Implications for biological structure. Chromosomes are giant nucleoprotein complexes in which the carrier of genetic information, DNA, is elaborately packaged by systematic, hierarchical winding about proteins called histones. In the first level of winding a stretch of about 145 DNA base pairs (bp) winds almost twice around a core of eight histones. The dimensions of the resulting structure, called the nucleosome core particle, are such that one may visualize the protein octamer as a squat cylinder with ends, about twice as wide as it is tall, with the DNA wound, like a piece of string, more or less helically on the cylindrical surface. The top and bottom discs of the cylinder are free of DNA, and the lateral surface is covered with DNA [3].

It may not be an accident that the nucleosomal length of $145 \mathrm{bp}$ is about the same as the persistence length of free DNA under near-physiological conditions (160 bp at $0.1 \mathrm{M}$ $\mathrm{NaCl}$ and $25^{\circ} \mathrm{C}$ ). The persistence length of any polymer gives the scale on which the polymer possesses elastic bending resiliency in the face of thermal buffeting by solvent [4]. Thus, nucleosomal DNA is not really like a piece of string; it is more like an elastic line, in the sense of this paper. For both the kinetics of formation of the nucleosome core particle and its final structure, the effects of the elastic resilience of DNA should be considered.

The critical length $l^{*}$ found above corresponds to about a 16 bp stretch of DNA. A stretch of DNA longer than 16 bp will deviate toward the axial direction, even if it starts winding circumferentially (i.e., with small pitch) around the histone core. The observed winding of nucleosomal DNA is almost circumferential. The natural tendency of DNA, due to its bending resilience, to deviate toward the axial direction as it winds would provide opportunity for an orderly progression during the winding process. In the absence of this effect, the bulky DNA polymer, on completing one turn, might collide with its starting point.

The tendency of an elastic line toward the axial direction is quite strong. According to Eq. 27, applied to the radial dimension of the nucleosome core particle, an infinitely long DNA polymer, winding on an infinitely long cylinder with initially circumferential 
direction, would require $2 \times 10^{6}$ bp to complete two turns around the cylinder. Of course, thermal buffeting would have destroyed the elastic resiliency of DNA long before such a length was reached, but, still, a comparison with $145 \mathrm{bp}$ of two turns of nucleosomal DNA is striking. In another view, the DNA polymer would complete only about half a turn after $145 \mathrm{bp}$, compared with two turns of $145 \mathrm{bp}$ of nuclesomal DNA.

Elongated protein structures are commonly found, microtubules, for example. If nature had at some point in evolutionary history tried to wind DNA on a long cylinder, she would quickly have learned of the uselessness of this packaging scheme and gone on to something else. The advantage of the small histone octamer could be that DNA, to avail itself of the negative energy of binding to the octamer surface, must wrap efficiently.

\section{REFERENCES}

[1] (i. S. Manning, Relaxed elastic line on a curved surface, Quart. Appl. Math. 45, 515-527 (1987)

[2] H. K. Nickerson and G. S. Manning, Intrinsic equations for a relaxed elastic line on an oriented surface, Geom. Dedicat. in press

[3] J. Darnell, H. Lodish, and D. Baltimore, Molecular cell biologv, Scientific American Books, New York, 1986

[4] (i. S. Manning. Polymer persistence length characterized as a critical length for instability caused by a fluctuating twist, Phys. Rev. A 34, 668-670 (1986) 